\title{
In vitro anti-cancer activity of a novel microbial fermentation product on human carcinomas
}

\author{
CHUNG HIN CHUI ${ }^{1,2}$, ROBERTO GAMBARI ${ }^{3}$, FUNG YI LAU ${ }^{2}$, GREGORY YIN MING CHENG $^{2}$, \\ RAYMOND SIU MING WONG ${ }^{2}$, STANTON HON LUNG KOK ${ }^{1}$, JOHNNY CHEUK ON TANG ${ }^{1}$, \\ IVY TUANG NGO TEO ${ }^{1,2}$, FILLY CHEUNG ${ }^{1,2}$, CHOR HING CHENG $^{1,2}$, KWOK PING HO ${ }^{1,2}$, \\ ALBERT SUN CHI CHAN ${ }^{1}$ and ALFONSO WONG ${ }^{4}$
}

\begin{abstract}
${ }^{1}$ Anti-Cancer Research Center, Central Laboratory of the Institute of Molecular Technology for Drug Discovery and Synthesis, State Key Laboratory of Chinese Medicine and Molecular Pharmacology, Department of Applied Biology and Chemical Technology, The Hong Kong Polytechnic University; ${ }^{2}$ Department of Medicine and Therapeutics, Prince of Wales Hospital, The Chinese University of Hong Kong, P.R. China; ${ }^{3}$ ER-GenTech, Department of Biochemistry and Molecular Biology, Section of Molecular Biology, University of Ferrara, Italy;

${ }^{4}$ International Naturopathic Cancer Research Institute, P.R. China
\end{abstract}

Received October 24, 2005; Accepted December 30, 2005

\begin{abstract}
The possible anti-proliferation and cell death induction potential of a novel microbial fermentation extract named as oncogen XP-180 (or simply as XP-180) was tested on three human solid tumour carcinoma cell lines (non-small cell lung cancer A549, breast cancer MDA-MB231, liver adenocarcinoma SK-Hep1) and on the acute myelogenous leukaemia KG1a cell line. Anti-proliferative activity of XP180 was observed on all of these cancer cell lines with comparable efficiency and in a dose-dependent manner. Morphological investigation further suggested that common features of apoptosis, including cell shrinkage and rounding, are present in XP-180 treated cells. Loss of adhesion properties of these solid tumour cell lines was observed upon XP-180 incubation. Anchorage-dependent clonogenicity assay on solid tumour cell lines and semi-solid methylcellulose colony formation assay on leukaemia cell line further revealed that XP-180 strongly inhibited the regeneration potential of these cancer cells. Using KG1a as an experimental model system, XP-180 was shown to stimulate the activity of caspase 3,8 and 9 without
\end{abstract}

Correspondence to: Professor Gregory Yin Ming Cheng, Department of Medicine and Therapeutics, Prince of Wales Hospital, The Chinese University of Hong Kong, Ngan Shing Street, Sha Tin, Hong Kong, P.R. China

E-mail: gcheng@cuhk.edu.hk

Dr Johnny Cheung On Tang, Department of Applied Biology and Chemical Technology, The Hong Kong Polytechnic University, Hung Hom, Kowloon, Hong Kong, P.R. China

E-mail: bccotang@inet.polyu.edu.hk

Key words: apoptosis, neoplasms, oncogen XP-180 significant change in caspase 6 activity. Furthermore, XP180 readily induced collapse of mitochondrial membrane potential after $2 \mathrm{~h}$ of incubation. However, the use of the generic caspase specific inhibitor Z-VAD-FMK does not significantly reverse XP-180 mediated cell death. The results obtained suggest that XP-180-mediated cancer cell death could involve mitochondria and both caspase-dependent and -independent pathways. Therefore, XP-180 is an efficient anti-cancer regimen in vitro.

\section{Introduction}

Cancer is still one of the most common fatal diseases in the human population. In Hong Kong, lung carcinoma is the first life-threatening pathology among carcinomas (1). Lung carcinoma should be considered with great attention from the epidemiological point of view, since both active and passive smokers are believed to have a higher incidence of getting this pathology than non-smokers (1). Another important neoplastic disease is breast cancer, more common in Western part of the world, but the rate is increasing in East Asia (2). The rate of occurrence of hepatocellular carcinoma (HCC) is relatively high in East Asia (1), in association with hepatitis B infection and liver cirrhosis $(3,4)$. Due to environmental pollution, leukaemic diseases are still very frequent (6). Thus, drugs exhibiting pharmacological activity against these forms of neoplasias are of great interest.

The study of microbial fermentation products as anticancer regimens is the object of great efforts, in addition to natural herbal medicines, as their qualities are under stringent control in both laboratory and industrial settings (7). We have demonstrated that the effective microogranism extract (EM-X) and Phaffia rhodozyma (Xanthophyllomyces denrorhous) extract fermented from brewer malt waste are active in vitro in growth inhibition and induction of cell death on several types of cancer cells (8-10). Both extracts possess strong antioxidative activity. Oncogen XP-180 is a novel microbial 
fermentation extract resulting from the biological activities of $>180$ strains of bacteria, including Lactobacillus Acidophilus, LC90-18 and Lactobacillus gasseri ATCC.

We screened for the possible anti-cancer potential of XP180 several solid tumours, including non-small cell lung cancer, breast cancer, hepatoma and acute myelogenous leukaemia (AML) cell lines. Our results suggest that XP-180 is an effective anti-proliferative and apoptosis inducing agent in the cancer cell lines tested. XP-180 significantly reduced the regeneration potential of cancer cells. Mitochondrion is involved while both caspase-dependent and -independent pathways are active in XP-180 induced apoptosis.

\section{Materials and methods}

Determination of concentration of XP-180. The original brownish fluid of XP-180 (kindly provided by Dr Alfonso Wong) was mixed with absolute ethanol. The mixture was then warmed in a round bottomed flask using a rotatory evaporator in a warm water bath. When all fluid was evaporated, the net weight of the dried extract was measured. The volume of fluid from each step was also recorded. The concentration of XP-180 extract was calculated to be approximately $24 \mathrm{mg} / \mathrm{ml}$.

Cell lines and cell culture condition. The cell lines employed in this study included the non-small cell lung cancer A549, the breast cancer MDA-MB231, the liver adenocarcinoma SK-Hep1 and the acute myelogenous leukaemia KG1a cell lines. All of them were obtained from American Type of Culture Collection. They were maintained routinely in minimum essential medium (MEM) (JRH BioSciences) supplemented with 5\% fetal bovine serum (Hyclone) together with penicillin and streptomycin (Invitrogen). Cell lines were kept in a $5 \%$ carbon dioxide humidified incubator at $37^{\circ} \mathrm{C}$.

Primary cultures from non-malignant haematological disordered bone marrow. After informed consent, bone marrow samples were obtained from three patients with non-malignant haematological disorder. Mononuclear cells were enriched and harvested immediately after Ficoll density centrifugation (Amersham, General Electricity) and washed with phosphate-buffered saline (PBS). Cell pellets were resuspended in RPMI-1640 culture medium and cell viability was determined by trypan blue exclusion assay.

Anti-proliferative and cytotoxic tests. The Cytolite chemiluminometric kit purchased from Perkin-Elmer was used to test the anti-proliferative and cytotoxic activites of XP-180. Briefly, different cells were seeded in a 96-well microtiter plate on the first day. On the second day, growth medium was changed and different concentrations of XP-180 [starting from $10 \%(\mathrm{v} / \mathrm{v})$ by volume ratio, i.e. $2.4 \mathrm{mg} / \mathrm{ml}$ ] was added. In each case, $10 \%(\mathrm{v} / \mathrm{v})$ of sterile water was used as control. Serial dilutions of XP-180 were performed in water [10\% (v/v) of final volume]. After $48 \mathrm{~h}$, the resulting luminescence values from water control and concentrated XP-180 treated cells were measured using a Victor ${ }^{2} \mathrm{~V}$ multilabel 1420 counter (Perkin-Elmer) according to the instruction manual provided.
Morphological changes of XP-180 treated cell. Cancer cells were seeded as mentioned before and then $10 \%(\mathrm{v} / \mathrm{v})$ of XP-180 was added. Any morphological changes after $48 \mathrm{~h}$ were determined under an inverted microscope $(11,12)$.

Colony formation assay of solid tumour cell lines. The cancer cell lines A549, MDA-MB231 and SK-Hep1 were incubated with $5 \%(\mathrm{v} / \mathrm{v})$ of XP-180 plus $5 \%(\mathrm{v} / \mathrm{v})$ sterile distilled water or $10 \%(\mathrm{v} / \mathrm{v}) \mathrm{XP}-180$ for 6 days. Untreated control cells were incubated with $10 \%(\mathrm{v} / \mathrm{v})$ of sterile distilled water only for 6 days. Culture plates were washed, then stained with methylene blue, washed again, and then photographed.

Colony formation assay of KGla and bone marrow cells. KG1a AML cells and mononuclear cells isolated from bone marrow samples were counted and cultured in methylcellulose (Stemcell Technology, Canada) with either vehicle or $10 \%$ (v/v) of XP-180 for 14 days. Viable colony formation units (CFU) [reducing 3-(4,5-dimethylthiazoyl)-2,5-diphenyltetrazolium bromide] (MTT) were then scored under an inverted microscope.

Caspase activity assay. To study whether caspase 3, 6 and 8 protease activities were increased after $24 \mathrm{~h}$ of XP-180 treatment on KG1a AML cells, we used a time resolved fluorescence technology based caspase 3, 6 and a caspase 8 activity kit purchased from Lance, Perkin-Elmer (Finland). Briefly, XP-180 treated cells were washed with PBS and then total cellular protein extracts were prepared and quantitated by the Bradford method using bovine serum albumin as calibration. Caspase 3, 6 and 8 specific substrates and detection buffer were added to equal amount of total cancer cell protein extract and individual caspase activity was analysed according to the instruction manual provided. The resulting relative fluorescence units were compared with untreated control (13). For caspase 9 activity, we used the Caspase-Glo 9 assay kit purchased from Promega. The assay procedure was performed as described before, except that caspase 9 activity was determined by a homogeneous luminescent assay.

Depolarization of the mitochondrial membrane potential. We used JC-1 as the detection probe (Molecular Probes) for the change of mitochondrial membrane potential. Briefly, after incubating KG1a AML cells with XP-180 for $2 \mathrm{~h}$, they were stained with JC-1. Mitochondria with a high membrane potential retained JC-1 as J-aggreate and gave a red signal. When there was depolarization of mitochondrial membrane potential, JC-1 was lost from the mitochondria and a shift from red to green resulted. Thus, the change of green/red fluorescence intensity ratio from low to high value indicated that there was a collapse in the mitochondrial membrane potential.

Caspase dependency cytotoxicity assay. To determine whether XP-180 mediated induction of apoptosis also involve caspaseindependent pathways, KG1a AML cells were pretreated with capase inhibitor Z-VAD-FMK, at concentration of $30 \mu \mathrm{M}$. Afterwards, either $5 \%(\mathrm{v} / \mathrm{v})$ or $10 \%(\mathrm{v} / \mathrm{v})$ of XP-180 was added and cancer cells were further incubated for $48 \mathrm{~h}$. The 

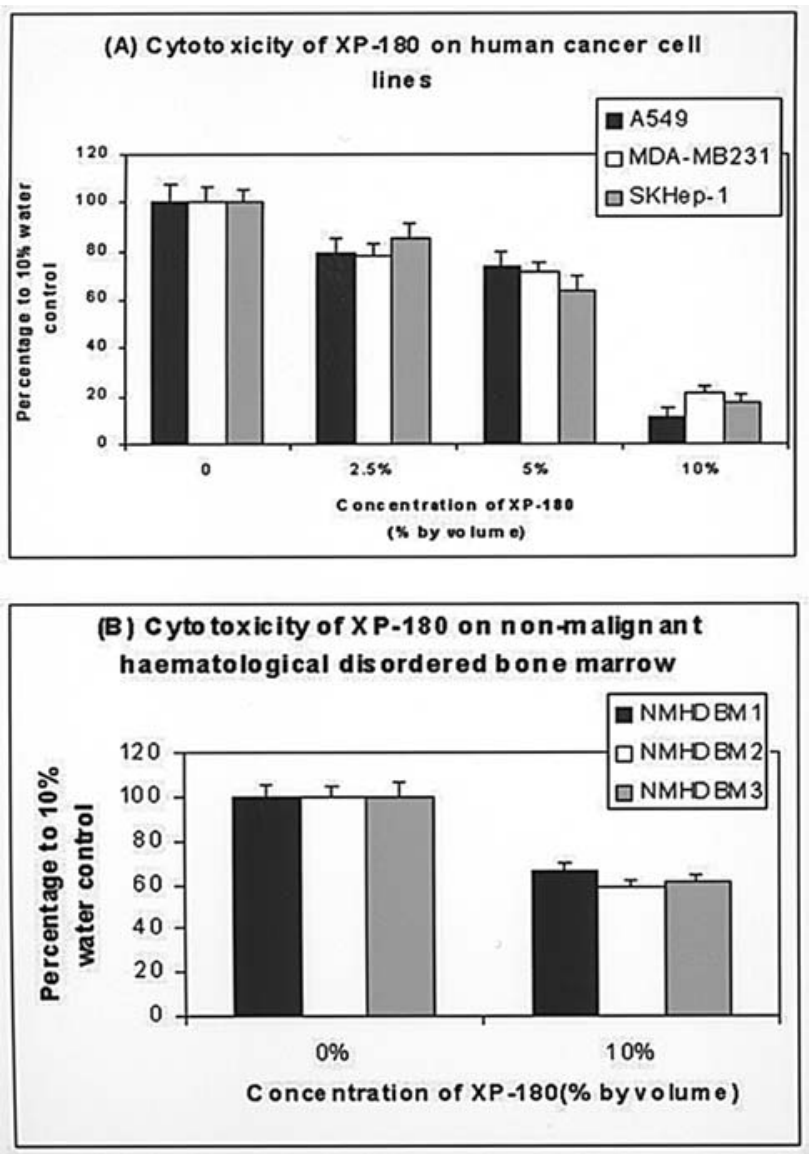

Figure 1. (A) In vitro anti-cancer potential of the fermentation extract XP-180 on different human solid tumour cancer cell lines. Cancer cells were incubated with different concentrations of XP-180 (v/v) for $48 \mathrm{~h}$ and total cellular survival was measured. Results are shown as mean $\pm \mathrm{SD}$ of triplicate tests from a single experiment, representative of three independent experiments leading to very similar results. White bar, A549 non-small cell lung cancer; black bar, MDA-MB231 breast cancer; grey bar, SK-Hep1 liver cancer. (B) Activity of XP-180 on non-malignant haematological disordered bone marrow cells. The conditions of treatment were the same as those employed for solid tumour cell lines. Results are shown as mean \pm SD of triplicate tests from a representative single experiment.

resulting cellular viability was then quantified by using the Cytolite kit.

\section{Results and Discussion}

Effects of XP-180 on cellular proliferation and cytotoxic activity. The results shown in Figs. $1 \mathrm{~A}$ and 2 clearly indicate that XP-180 effectively exerts anti-proliferative and cytotoxic effects on A549 lung cancer, MDA-MB231 breast cancer and SK-Hep1 hepatoma cell lines. The effects were dose dependent. A maximum cytotoxic activity was found at $10 \%$ (v/v), experimental conditions at which XP-180 exerts about $70-80 \%$ of cytotoxicity on the three tumour cell lines (Fig. 1A). Characteristic features of cell death, including cell shrinkage, rounding and loss of adhesion potential were observed (Fig. 2). On the other hand, only about a $40 \%$ growth inhibitory effect on three samples of non-malignant haematological disordered bone marrow could be detected at $10 \%(\mathrm{v} / \mathrm{v})$ of XP-180 (Fig. 1B).
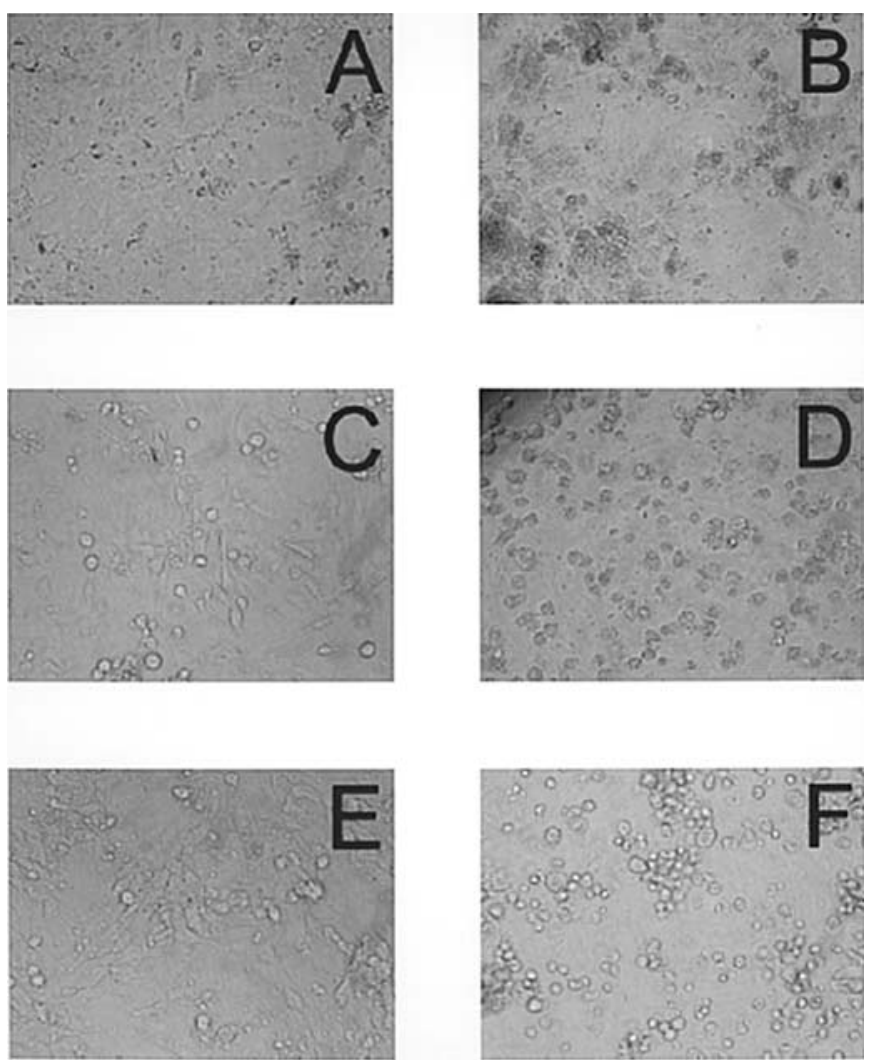

Figure 2. Morphological investigation under inverted microscope of cell shrinkage, loss of colony formation ability and adherent potential of the three solid tumour cancer cell lines treated with XP-180 (10\% by volume) for $48 \mathrm{~h}$. (A and B) A549 non-small cell lung cancer, (C and D) MDA-MB231 breast cancer and lastly, (E and F) SK-Hep1 liver cancer. Cells in (A), (C) and (E) were treated with $10 \%(\mathrm{v} / \mathrm{v})$ vehicle while cells in (B), (D) and (F) were treated with $10 \%(\mathrm{v} / \mathrm{v})$ of XP-180.

Effects of XP-180 on colony formation potential. After incubation of the solid tumour and KG1a AML cells with XP-180, we analysed the effects of XP-180 on their colony formation potential. The results obtained demonstrated that concentrated XP-180 is able to effectively reduce their regeneration potential. A dose-dependent relationship (from $1.22 \mathrm{mg} / \mathrm{ml}$ to $2.44 \mathrm{mg} / \mathrm{ml}$ ) was found in the case of A549, MDA-MB231 and SK-Hep1 cancer cells (Fig. 3A-C). At the dosage of $10 \%(\mathrm{v} / \mathrm{v})(2.44 \mathrm{mg} / \mathrm{ml})$, complete loss of colony formation was observed for all the solid tumour cell lines employed (Fig. 3A-C). In the case of KG1a, a complete inhibition on the regeneration potential was also found at the dose of $10 \%$ (v/v) of XP-180 (Fig. 3D).

When the experiments were performed on primary cultured bone marrow cells, concentrated XP-180 was found to reduce also the regeneration potential of bone marrow cells isolated from patients with non-malignant haematological disorders (Fig. 3D, lower pair). However, the degree of inhibition of colony formation when bone marrow cells isolated from patients with non-malignant haematological disorders were employed was not as strong as the inhibition observed with KG1a AML cells.

Activity of caspase 3, 6, 8 and 9 following XP-180 treatment. After demonstrating that XP-180 was active on cancer cell lines, we focused our experiments on the investigation of 

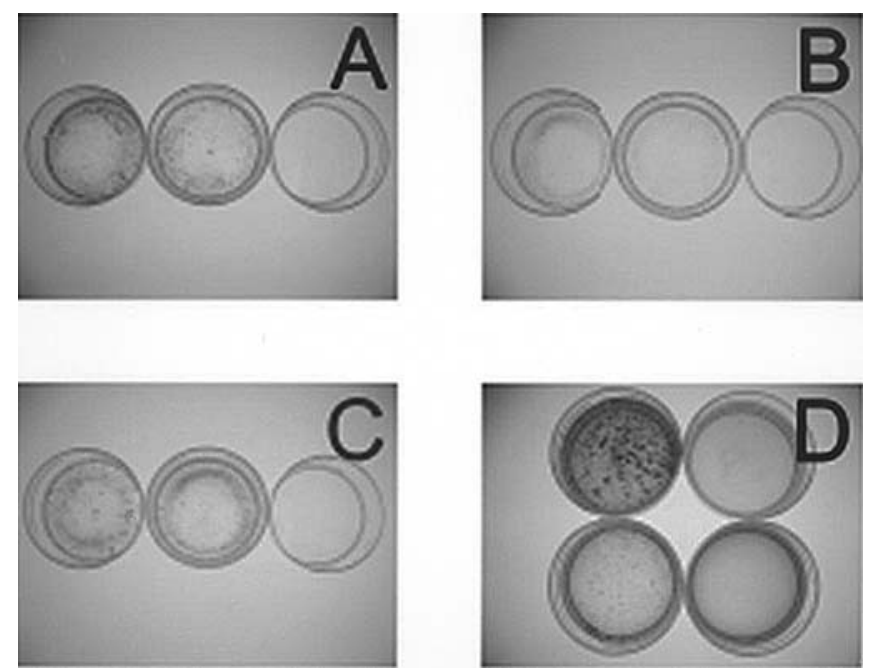

Figure 3. Effects of XP-180 on the regeneration potential of (A) A549 lung cancer, (B) MDA-MB231 breast cancer and (C) SK-Hep1 liver adenocarcinoma cell lines using anchorage-dependent clonogenic assay. For each panel: left, vehicle only; middle, 5\% (v/v) XP-180; right, $10 \%$ (v/v) XP-180. (D) Effects of XP-180 on the regeneration potential of KG1a leukaemia cell line as well as non-malignant haematological disorder bone marrow using semi-solid methylcellulose clonogenic assay. Left, vehicle only; right, $10 \%$ (v/v) XP-180. The upper pair refers to KG1a cells while the lower pair is non-malignant haematological disorder bone marrow. Representative results of three independent experiments leading to similar results are shown.

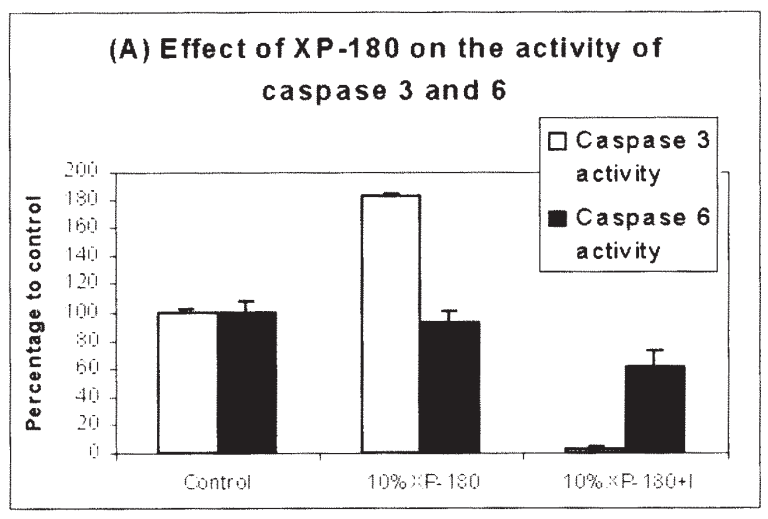

(B) Effect of XP-180 on the a ctivity of c aspase 8 and 9

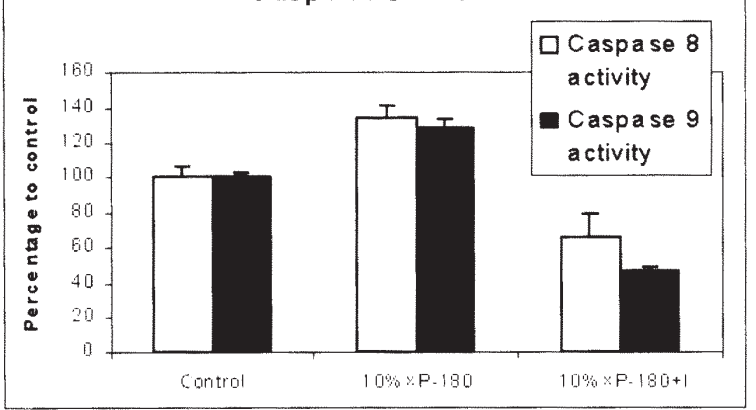

Figure 4. Activity of caspases 3 and 6 (A) and caspase 8 and 9 (B) on XP-180 treated KG1a cells. Results represent the average \pm SD of triplicate tests from one experiment, representative of three independent experiments, leading to similar results. Results obtained using XP-180 in the presence of the caspase inhibitor Z-VAD-FMK (indicated as 'I') are shown on the right in both (A) and (B).
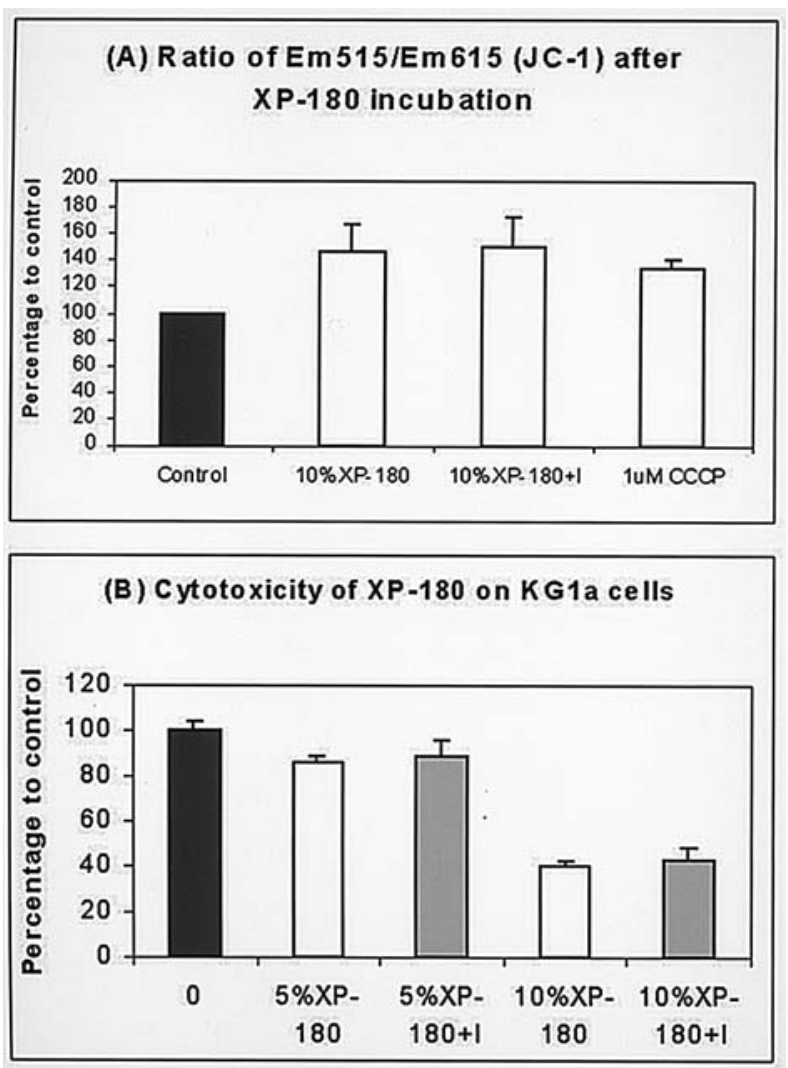

Figure 5. (A) XP-180 induced depolarization of the mitochondrial membrane potential in AML KG1a cells after staining with JC-1 probe. An increment of emission $515 \mathrm{~nm}$ to emission 615 after an excitation at $485 \mathrm{~nm}$ indicated that there was a collapse in mitochondrial membrane potential. CCCP is an ionophore able to cause a dissipation of the proton gradient built up on the inner mitochondrial membrane and used as a positive control. (B) Cytotoxicity assay on XP-180 treated KG1a cells after $48 \mathrm{~h}$ of incubation in the absence or presence of the caspase inhibitor Z-VAD-FMK (indicated as 'I'). Each experiment was done in triplicate. Results represent the average of three independent experiments.

possible mechanism(s) of action of XP-180, using the AML KG1a cell line as the experimental model system. Since caspases are probably the most important effector molecules triggering apoptosis, we attempted to determine whether XP180 is able to activate endogenous caspase activity at a concentration of $10 \%(\mathrm{v} / \mathrm{v})$. The results obtained demonstrate that caspase 3 was significantly activated while no significant changes in the proteolytic activity mediated by caspase 6 was detected (Fig. 4A).

Caspase 8 and 9 are important for cascade activation of downstream capase activity. After $10 \%$ (v/v) XP-180 stimulation, both caspase 8 and 9 were found to be activated. Thus our results suggested that XP-180 first activated caspase 8 and 9 , which in turn stimulated caspase 3 and leading to apoptosis of tumour cells (Fig. 4B).

$X P-180$ induces depolarization of the mitochondrial membrane potential. Since caspase 9 activity is associated with XP-180 induced apoptosis, it was highly possible that mitochondria are also involved. Thus, we tested whether there was any collapse in mitochondrial membrane potential in XP-180 treated cells. As shown in Fig. 4A, XP-180 at 10\% $(\mathrm{v} / \mathrm{v})$ readily enhanced mitochondrial membrane collapse. 
Co-incubation with caspase inhibitor Z-VAD-FMK could not protect mitochondria from depolarization (Fig. 5A). Therefore, it is possible that mitochondrial alterations are essential for the action of XP-180.

Caspase 3-independent pathways are also involved in XP-180mediated apoptosis. Since we demonstrated that caspase 3,8 and 9 were stimulated by XP-180, we determined whether the block of induction of these apoptotic mediators could inhibit cell death. Using the Cytolite cytotoxicity kit, we analyzed whether the caspase inhibitor Z-VAD-FMK could reverse the action of XP-180 on AML KG1a cells. As shown in Fig. 5B, the presence of caspase inhibitor at a concentration effectively suppressing the XP-180 mediated increase of caspase activity (right side of Fig. 4) could not significantly reverse the action of XP-180 when used at either $5 \%(\mathrm{v} / \mathrm{v})$ or $10 \%(\mathrm{v} / \mathrm{v})($ Fig. 5B). Therefore, we concluded that both caspase-dependent and -independent pathways are active in XP-180 mediated apoptosis.

Many naturally occurring products are shown to be useful in cancer treatment. These include many traditional Chinese herbal medicines such as anomalous fruit extract of Gleditsia sinensis (14), and extracts of Scutellaria barbata and Radix Sophorae Tonkinensis (15), Brucea javanica (16) and Salvia mithiorritza (17). Moreover, green tea, derived from Camellia sinensis is considered an anti-cancer preventing agent (18). As alternative cancer therapy becomes more recognized and acceptable, the uses of microbial fermentation products and herbal medicines should provide cancer patients a supplementary treatment in the foreseeable future, in addition to conventional chemotherapy. The results herein reported support the concept that anti-cancer agents can be reproducibly found in natural products (19).

\section{Acknowledgements}

We acknowledge the Hong Kong Polytechnic University (Area of Strategic Development Fund, HKPU), the University Grants Committee (Areas of Excellence Scheme) of the Hong Kong (Project No. AoE/P-10/01) and a Niche Area grant form HKPU. Appreciation is given to Bio-gene Technology Co. Ltd. and Classic Trend Investment Ltd. for their donation to Haematology Research Funding in Haematology Division, Department of Medicine and Therapeutics, Prince of Wales Hospital (PWH), The Chinese University of Hong Kong (CUHK). Drs F.Y.L. and C.H.C. are the honourary tutors kindly offered by Professor Joseph J.Y. Sung and Professor G.Y.M.C. from Department of Medicine and Therapeutics, PWH, CUHK. Dr C.H.C. was supported by the post of 'Scientific Officer' offered by Professors K.Y. Wong and A.S.C.C. Professor R.G. is granted by AIRC and by UE (Interventi Strutturali Obiettivo 2).

\section{References}

1. Kit LW: Why is lung cancer common in non-smoking women in Hong Kong? From epidemiologic to molecular studies. Chin Med J 110: 420-424, 1997

2. Wu TY, Bancroft J and Guthrie B: An integrative review on breast cancer screening practice and correlates among Chinese, Korean, Filipino, and Asian Indian American women. Health Care Women Int 26: 225-246, 2005.
3. Kao JH and Chen DS: Changing disease burden of hepatocellular carcinoma in the Far East and Southeast Asia. Liver Int 25: 696-703, 2005.

4. Cheung J, Lee TK, Teh CZ, Wang CY, Kwan WC and Yoshida EM: Cross-sectional study of hepatitis B awareness among Chinese and Southeast Asian Canadians in the Vancouver-Richmond community. Can J Gastroenterol 19: 245$249,2005$.

5. Fattovich G, Stroffolini T, Zagni I and Donato F: Hepatocellular carcinoma in cirrhosis: incidence and risk factors. Gastroenterology 27: S35-S50, 2004.

6. Barletta E, Gorini G, Vineis P, Miligi L, Davico L, Mugnai G, Ciolli S, Leoni F, Bertini M, Matullo G and Costantini AS: Ras gene mutations in patients with acute myeloid leukaemia and exposure to chemical agents. Carcinogenesis 25: 749-755, 2004.

7. Chui CH, Gambari R, Lau FY, Teo ITN, Ho KP, Cheng GYM, Ke B, Higa T, Kok SHL, Chan ASC and Tang JCO: Anti-cancer potential of traditional Chinese herbal medicines and microbial fermentation products. Minerva Biotecnol 17: 183-192, 2005.

8. Chui CH, Cheng GYM, Ke B, Lau FY, Wong RSM, Kok SHL, Fatima S, Cheung F, Cheng CH, Chan ASC and Tang JCO: Growth inhibitory potential of effective microorganism fermentation extract (EM-X) on cancer cells. Int J Mol Med 14: 925-929, 2004.

9. Teo ITN, Chui CH, Tang JCO, Lau FY, Cheng GYM, Wong RSM, Kok SHL, Cheng CH, Chan ASC and Ho KP: Antiproliferation and induction of cell death of Phaffia rhodozyma extract fermented by brewer malt waste on breast cancer cells. Int J Mol Med 16: 931-936, 2005.

10. Chow LMC, Chui CH, Tang JCO, Teo I, Lau FY, Cheng GYM, Wong RSM, Leung TWT, Lai KB, Yau M, Gou D and Chan ASC: Gleditsia sinensis fruit extract is a potential chemotherapeutic agent in chronic and acute myelogenous leukemia. Oncol Rep 10: 1601-1607, 2003.

11. Chui CH, Tang JCO, Lau FY, Teo ITN, Yau MYC, Wong RSM, Cheng GYM, Ho SKW, Leung TWT, Hui KS, Wong MM, Fatima S, Cheng CH, Cheung F, Tan WQ, Chow LMC, Guo D and Chan ASC: Gleditsia sinensis fruit extract induced growth inhibition involved basic fibroblast growth factor and nitric oxide. Int J Mol Med 13: 169-173, 2004.

12. Teo ITN, Tang JCO, Chui CH, Cheng GYM, Yau MYC, Lau FY, Wong RSM, Leung TWT, Cheung F, Ho KP, Cheng CH and Chan ASC: Superoxide anion is involved in the early apoptosis mediated by Gleditsia sinensis fruit extract. Int J Mol Med 13: 909-913, 2004.

13. Chui CH, Lau FY, Chan ASC, Cheng GYM, Wong RSM, Lai KB, Kok SHL, Au Yeung TTL, Teo ITN, Yau MYC, Cheung F, Cheng CH and Tang JCO: Gleditsia sinensis fruit extract-induced apoptosis involves changes of reactive oxygen species level, mitochondrial membrane depolarization and caspase 3 activation. Int J Mol Med 15: 539-543, 2005.

14. Cheung F, Chui CH, Chan ASC, Lau FY, Cheng GYM, Wong RSM, Kok SHL, Teo ITN, Cheng CH and Tang JCO: Inhibition of proteasome activity in Gleditsia sinensis fruit extract-mediated apoptosis on human carcinoma cells. Int J Mol Med 16: 925-929, 2005.

15. Chui CH, Lau FY, Tang JCO, Kan KL, Cheng GYM, Wong RSM, Kok SHL, Lai PBS, Ho R, Gambari R and Chan ACS: Activities of fresh juice of Scutellaria barbata and warmed water extract of Radix Sophorae Tonkinensis on anti-proliferation and apoptosis of human cancer cell lines. Int J Mol Med 16: 337-341, 2005.

16. Lau FY, Chui CH, Gambari R, Kok SHL, Kan KL, Cheng GYM, Wong RSM, Teo ITN, Cheng CH, Wan TSK, Chan ASC and Tang JCO: Antiproliferative and apoptosis-inducing activity of Bruces javanica extract on human carcinoma cells. Int $\mathrm{J}$ Mol Med 16: 1157-1162, 2005.

17. Guo X, Gao W and Xiao P: Factors affecting root growth and metabolite production sqin Salvia miltiorrhiza adventitious root cultures. Minerva Biotecnol 17: 133-140, 2005.

18. Farabegoli F: Green tea in human cancer. Minerva Biotecnol 17: 163-174, 2005.

19. Hollosy F and Keri G: Plant-derived protein tyrosine kinase inhibitors as anticancer agents. Curr Med Chem Anti-Cancer Agents 4: 173-197, 2004. 